MPE - Volume 2, pp. 199-204

Reprints available directly from the publisher Photocopying permitted by license only
(C) 1996 OPA (Overseas Publishers Association) Amsterdam B.V. Published in The Netherlands under license by Gordon and Breach Science

Publishers SA

Printed in Malaysia

\title{
ANALYSIS OF NONLINEAR SYSTEMS WITH TIME VARYING INPUTS AND ITS APPLICATION TO GAIN SCHEDULING
}

\author{
J.-T. LIM and S.-K. CHO \\ Department of Electrical Engineering, Korea Advanced Institute of Science and \\ Technology, 373-1, Kuseng-dong, Yusung-gu, Taejon, 305-701, Korea
}

(Received 1 March 1995; in final form 11 June 1995)

\begin{abstract}
An analytical framework for analysis of a class of nonlinear systems with time varying inputs is presented. It is shown that the trajectories of the transformed nonlinear systems are uniformly bounded with an ultimate bound under certain conditions shown in this paper. The result obtained is useful for applications, in particular, analysis and design of gain scheduling.
\end{abstract}

AMS No.: $93 \mathrm{C} 10$

KEYwORDS: Gain scheduling; nonlinear system; time varying input

\section{INTRODUCTION}

The main idea of gain scheduling is that linear design methods are applied to nonlinear control design provided that the scheduling variable captures the plant's nonlinearity and varies slowly. In spite of its wideapplications [1]-[3], the analytical framework on analysis and design of gain scheduling is still in process [4]-[8] to give the gain scheduling approach a rigorous mathematical justification. Based on the Gronwall-Bellman inequality, a stability theorem has been presented in [4]. In [5]-[8], Liapunov functions have been constructed to investigate the robustness and stability properties. However most of their results are limited to slow variations in the scheduling variables. In this paper, we present a theoretical foundation for gain scheduling without restriction to slow variations in the scheduling variables. We use Liapunov stability to analyze a class of nonlinear systems with time varying inputs and obtain an ultimate bound on the discrepancy between the exact solution and the fixed operating point. Since gain scheduling is a nonlinear feedback whose parameters evolve as functions of exogeneous variables, gain scheduling yields a closed loop system that is nonlinear, at least in the scheduling variables. Thus the ultimate behavior of the resulting nonlinear system with time varying inputs can be analyzed using the Theorem formulated below.

\section{MAIN RESULT}

Consider the following system. 


$$
\dot{x}=f(x, u(t))
$$

where $x \in R^{n}$ and $u(t) \in \Gamma$ which is a bounded, open subset of $R^{m}, \forall t \geq 0$. Let $\|\cdot\|$ denote the Euclidean norm of a time varying vector, and also the corresponding induced norm on a matrix. Assume that

(i) $f$ is twice continuously differentiable,

(ii) the equation $f(x, u(t))=0, \forall u(t) \in \Gamma$ has a continuously differentiable solution $x=h(u)$,

(iii) $\|\dot{u}(t)\|<\gamma_{1}, \forall t \geq 0$,

(iv) there exist positive constants $\sigma, k_{1}$, and $k_{2}$ such that $\lambda_{i}(u(t))+\lambda_{i}^{*}(u(t)) \leq-2 \sigma, k_{1} \sigma$ $\leq\left|\lambda_{i}(u(t))\right| \leq k_{2} \sigma, \forall u(t) \in \Gamma, i=1, \ldots, n$ where $\lambda_{i}(u(t))$ is the eigenvalue of $\frac{\partial f}{\partial x}(h(u), u(t))$ for each fixed $t \geq 0$ and $\lambda_{i}^{*}(u(t))$ is the complex conjugate of $\lambda_{i}(u(t))$.

Since $f$ is continuously differentiable, $f(x, u(t))$ can be rewritten as

$$
\begin{aligned}
f(x, u(t))= & f(h(u), u(t))+\frac{\partial f}{\partial x}(h(u), u(t))(x-h(u)) \\
& +\left[\frac{\partial f}{\partial x}\left(x^{\prime}, u(t)\right)-\frac{\partial f}{\partial x}(h(u), u(t))\right](x-h(u))
\end{aligned}
$$

where $x^{\prime}$ is a point on the line segment joining $x$ and $h(u)$. Let $y=x-h(u)$, then

$$
\dot{y}=f(x, u)-\frac{\partial h(u)}{\partial u} \dot{u}(t)
$$

Using the fact that $\frac{\partial f}{\partial t}(h(u), u(t))=0$, we have $\frac{\partial f}{\partial x}(h(u), u(t)) \frac{\partial h(u)}{\partial u} \dot{u}(t)+\frac{\partial f}{\partial u}(h(u)$, $u(t)) \dot{u}(t)=0$. Since $\operatorname{det}\left[\frac{\partial f}{\partial x}(h(u), u(t))\right] \neq 0, \forall u(t) \in \Gamma$, we obtain

$$
\frac{\partial h(u)}{\partial u} \dot{u}(t)=-\left[\frac{\partial f}{\partial x}(h(u), u(t))\right]^{-1} \frac{\partial f}{\partial u}(h(u), u(t)) \dot{u}(t)
$$

From (1)-(4),

$$
\dot{y}=A(t) y+R(t) y+B(t) \dot{u}
$$

where $A(t)=\frac{\partial f}{\partial x}(h(u), u(t)), R(t)=\left[\frac{\partial f}{\partial x}\left(x^{\prime}, u(t)\right)-\frac{\partial f}{\partial x}(h(u), u(t))\right]$, and $B(t)=$ $\left[\frac{\partial f}{\partial x}(h(u), u(t))\right]^{-1} \frac{\partial f}{\partial u}(h(u), u(t))$. 
Theorem: Consider the system (1) with assumptions (i)-(iv). Then there exists a positive constant $\sigma_{o}$ such that, for any $\sigma>\sigma_{o}$,

$$
\|y(t)\| \leq \eta\|y(0)\| e^{-\alpha_{1}(\sigma) t}+\alpha_{2}(\sigma), \forall\|y(0)\|<\alpha_{3}(\sigma)
$$

where $\eta$ is a positive constant and $\alpha_{I}(\cdot), \alpha_{2}(\cdot), \alpha_{3}(\cdot)$ are class- $K$, class- $L$, class- $K$ functions (see [8]), respectively.

The proof of Theorem requires two auxiliary results.

LEMMA 1: Under assumptions (i)-(iv), let $Q(t)$ be a bounded matrix which is defined by

$$
Q(t)=\int_{0}^{\infty} e^{A^{T}(t) \tau} e^{A(t) \tau} d \tau
$$

where $A(t)=\frac{\partial f}{\partial x}(h(u), u(t))$ and let $\sigma$ be a positive constant given in the assumption (iv). Then there exist positive constants $c_{1}, c_{2}, c_{3}$ such that $\|A(t)\| \leq c_{1} \sigma,\left\|A^{-1}(t)\right\| \leq \frac{c_{2}}{\sigma}$, $\|Q(t)\| \leq \frac{c_{3}}{\sigma}$.

Proof: Let $A_{o}(t)=\frac{1}{\sigma} A(t)$, then there exists a nonsingular matrix $P(t)$ such that

$$
A(t)=\sigma P^{-1}(t) A_{J}(t) P(t)
$$

where $A_{J}(t)$ is the Jordan form representation of $A_{o}(t)$. Since $A(t)$ is bounded, there exists a positive constant $k_{a}$ such that $\|A(t)\| \leq k_{a}$ o $\left\|A_{J}(t)\right\|$. Note that $\left\|A_{J}(t)\right\| \leq$ $\sqrt{\max _{i} \sum_{j=1}^{n}\left|\left(A_{J}(t)\right)_{i, j}\right| \max _{j} \sum_{i=1}^{n}\left|\left(A_{J}(t)\right)_{i, j}\right|}$. Let $n_{s}$ be the order of the largest Jordan block in $A_{J}(t)$. Then, by simple algebraic manipulations, we have $\left\|A_{J}(t)\right\| \leq k_{2}$ if $n_{s}=1$ and $1+$ $k_{2}$ otherwise. Thus $\|A(t)\| \leq k_{a} \sigma k_{2}$ if $n_{s}=1$ and $k_{a} \sigma\left(1+k_{2}\right)$ otherwise.

From (7), $A^{-1}(t)=\frac{1}{\sigma} P^{-1}(t) A_{J}^{-1}(t) P(t)$. Since $A^{-1}(t)$ is also bounded, there exists a positive constant $k_{b}$ such that $\left\|A^{-1}(t)\right\| \leq \frac{k_{b}}{\sigma}\left\|A_{J}^{-1}(t)\right\|$. Based on the same way, we obtain $\left\|A_{J}^{-1}(t)\right\| \leq \sum_{i=1}^{n_{s}} k_{1}^{-i}$. Thus $\left\|A^{-1}(t)\right\| \leq \frac{k_{b}}{\sigma} \sum_{i=1}^{n_{s}} k_{1}^{-i}$.

Again, from (7), $e^{A(t) \tau}=P^{-1}(t) e^{\sigma A_{J}}(t) \tau \rightarrow e^{\sigma A(t) \tau} P(t)$. Since $e^{A(t) \tau}$ is also a bounded matrix, there exists a positive constant $k_{c}$ such that $\left\|e^{A(t) \tau}\right\| \leq k_{c} \| e^{\sigma A_{J}}(t) \tau \rightarrow e^{\sigma A_{j}(t) \tau}$. In the same manner, we obtain $\left\|e^{\sigma A_{j}(t) \tau}\right\| \leq e^{-\sigma \tau} \sum_{i=1}^{n_{s}} \frac{(\sigma \tau)^{i-1}}{(i-1) !}$. Thus we have $\|Q(t)\| \leq$ $k_{c}^{2} \int_{0}^{\infty}\left\|e^{\sigma A_{j}(t) \tau}\right\|^{2} d \tau \leq \frac{k_{c}^{2}}{2 \sigma} \sum_{i=1}^{n_{s}} \sum_{j=1}^{n_{s}} \frac{(i+j-2) !}{2^{i+j-2}(i-1) !(j-1) !}$.

Q.E.D. 
Since $\frac{\partial f}{\partial x}(h(u), u(t)), \forall u(t) \in \Gamma$ is continuously differentiable, it follows that there exists a positive constant $\gamma_{2}$ such that $\|\dot{A}(t)\| \leq \gamma_{2}\|\dot{u}(t)\|, \forall t \geq 0$ where $A(t)=\frac{\partial f}{\partial x}(h(u)$, $u(t)$ ). Then we present the following lemma (see [6] for details) that will be used in the proof of Theorem.

LEMMA 2: Given $\|Q(t)\| \leq \frac{c_{3}}{\sigma},\|A(t)\| \leq c_{1} \sigma$, and $\|\dot{A}(t)\| \leq \gamma_{1} \gamma_{2}$ where $Q(t)$ and $A(t)$ are defined in Lemma 1, then the following relationships hold: (i) $A^{T}(t) Q(t)+Q(t) A(t)=-I$, (ii) $\frac{1}{2 c_{1} \sigma}\|y\|^{2} \leq y^{T} Q(t) y \leq \frac{c_{3}}{\sigma}\|y\|^{2}$,(iii) $\|\dot{Q}(t)\| \leq 2\left(\frac{c_{3}}{\sigma}\right)^{2} \gamma_{1} \gamma_{2}$.

Proof of the Theorem: Consider $V(t, y)=y^{T} Q(t) y$. Then, from (5) and Lemmas $1 \& 2$,

$$
\begin{aligned}
V(t, y)= & -y^{T}\left(A^{T}(t) Q(t)+Q(t) A(t)\right) y+y^{T} \dot{Q}(t) y \\
& +2 y^{T} Q(t) R(t) y+2 y^{T} Q(t) B(t) \dot{u} \\
\leq & -\|y\|^{2}+\|\dot{Q}(t)\|\|y\|^{2}+2\|Q(t)\|\|R(t)\|\|y\|^{2} \\
& +2\|Q(t)\|\|B(t)\|\|y\|\|\dot{u}\|
\end{aligned}
$$

Since $f(x, u(t))$ is twice continuously differentiable, $\frac{\partial f}{\partial x}(x, u(t))$ is Lipschitz in $x$ on any bounded region $B \in R^{n}$. Thus there exists a positive constant $L$ such that $\left\|\frac{\partial f}{\partial x}(x, u(t))-\frac{\partial f}{\partial x}(h(u), u(t))\right\| \leq L\|x-h(u)\|, \forall x, h(u) \in B, \forall u(t) \in \Gamma$. As it follows from (5), since $f(h(u), u(t)), \forall u(t) \in \Gamma$ is continuously differentiable, there exists a positive constant $\gamma_{3}$ such that $\|B(t)\| \leq \gamma_{3}\left\|A^{-1}(t)\right\|, \forall u(t) \in \Gamma$. Then, from Lemmas $1 \& 2$, it is easy to show

$$
\begin{aligned}
V(t, y) & <-\left(\frac{1}{2}-\frac{2 c_{3}^{2} \gamma_{1} \gamma_{2}}{\sigma^{2}}\right)\|y\|^{2}+\frac{2 c_{2} c_{3} \gamma_{1} \gamma_{3}}{\sigma^{2}}\|y\|, \forall\|y\|<\frac{\sigma}{4 c_{3} L} \\
& \leq-(1-\theta)\left(\frac{1}{2}-\frac{2 c_{3}^{2} \gamma_{1} \gamma_{2}}{\sigma^{2}}\right)\|y\|^{2}, \forall y \in D_{1}, 0<\theta<1
\end{aligned}
$$

where $D_{1}=\left\{y \in R^{n}: d_{2}<\|y\|<d_{1}\right\}$ and $d_{1}=\frac{\sigma}{4 c_{3} \mathrm{~L}}, d_{2}=\frac{4 c_{2} c_{3} \gamma_{1} \gamma_{3}}{\theta\left(\sigma^{2}-4 c_{3}^{2} \gamma_{1} \gamma_{2}\right)}$.

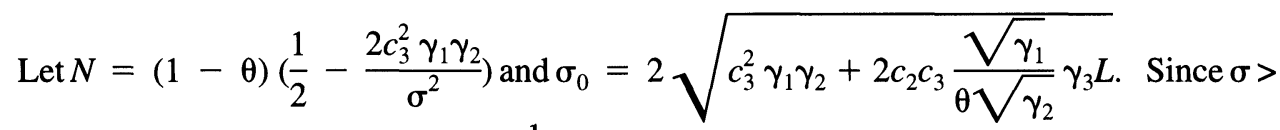
$\sigma_{0}$, it is easy to verify that $0<N<\frac{1}{2}$ and $D_{1} \neq \varnothing$. From Lemma 2 and (8), we obtain 


$$
V(t, y) \leq-\frac{\sigma N}{c_{3}} V(t, y), \forall y \in D_{1}
$$

Then, from Lemma 2 and (9),

$$
\|y(t)\|^{2} \leq 2 c_{1} \sigma V(0, y(0)) e^{-\frac{\sigma N t}{c_{3}}} \leq 2 c_{1} c_{3}\|y(0)\|^{2} e^{-\frac{\sigma N t}{c_{3}}}, \forall y \in D_{1}
$$

Hence $\|y(t)\| \leq \sqrt{2 c_{1} c_{3}}\|y(0)\| e^{-\frac{\sigma N t}{2 c_{3}}}, \forall y \in D_{1}$. Thus, by simple algebraic manipulations, we have

$$
\|y(t)\| \leq \sqrt{2 c_{1} c_{3}}\|y(0)\| e^{-\frac{\sigma N t}{2 c_{3}}}+\frac{4 c_{2} c_{3} \gamma_{1} \gamma_{3}}{\theta\left(\sigma^{2}-4 c_{3}^{2} \gamma_{1} \gamma_{2}\right)}, \forall y(0) \in D_{2}
$$

where $D_{2}=\left\{y(0) \in R^{n}:\|y(0)\|<\frac{d_{1}-d_{2}}{\sqrt{2 c_{1} c_{3}}}\right\}$.

Note that, for the fast varying $u(t)$, the ultimate bound in (10) can be made as small as possible by introducing large $\sigma$. If, in addition, $\lim _{t \rightarrow \infty} \dot{u}(t)=0$, then we can show $\lim _{t \rightarrow \infty} y(t)=0$ by repeating the steps of the proof of Theorem.

Example: Consider the following system with the desired output $c(t)=0$.

$$
\begin{aligned}
& \dot{x}_{1}(t)=-30 x_{1}(t)+x_{2}^{2}(t)+\sin 2 t \\
& \dot{x}_{2}(t)=x_{1}^{2}(t)+x_{2}(t)+u_{c}(t) \\
& c(t)=x_{2}(t)
\end{aligned}
$$

where $u_{c}(t)$ is the control input. Since the operating condition is specified by the exogeneous variable, $\sin 2 t$, it is used as a scheduling variable. For each fixed scheduling variable, the frozen system has a fixed operating point defined by $x=h(u)$. In order to have the desired output as fixed operating points, $h(u)=\left[\frac{\sin 2 t}{30} 0\right]^{T}$ and the gain scheduled control law is $u_{c}(t)=l x_{2}(t)-\left(\frac{\sin 2 t}{30}\right)^{2}$ where $l$ is the constant to be chosen. Since, as it follows from the above, $f(x, u(t))$ and $h(u)$ are smooth and the eigenvalues of $\frac{\partial f}{\partial x}(h(u), u(t))$ for each fixed $t \geq 0$ have negative real parts for any value of $l$ less than -1 , the conditions (i)-(ii) and (iv) are satisfied. It also follows from $u(t)=\sin 2 t$ that $u(t)$ satisfies the condition (iii). Thus all the conditions of Theorem are met and as it follows from (10), the ultimate bound on $y(t)$ is less than 0.007 for $l \leq-25$. Fig. 1 shows the trajectories of $x(t), y(t)$ for $l=-25$. It is observed from Fig. 1 that $x(t)$ asymptotically follows $h(u)$ and $y(t)$ is uniformly bounded with the ultimate bound obtained from (10). 

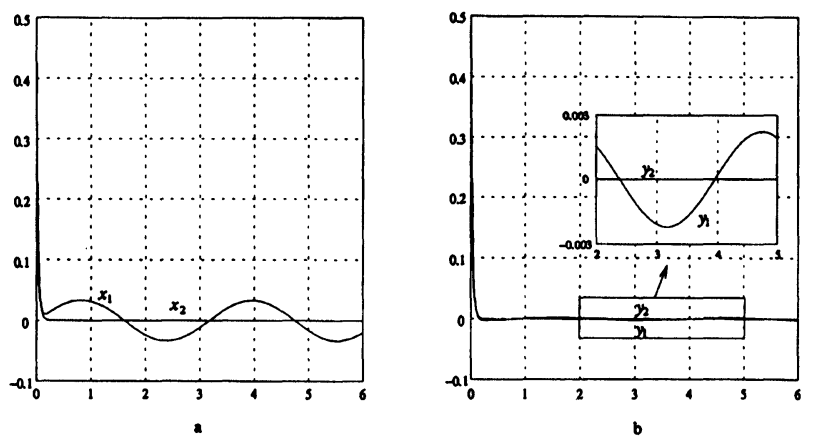

Figure 1 Asymptotic tracking performance

a Trajectory of $\mathrm{x}(t)$ for $1=-25$ with $\mathrm{x}(0)=\left[\begin{array}{ll}0.5 & 0.2\end{array}\right]^{T}$

b Trajectory of $\mathrm{y}(t)$ for $1=-25$ with $\mathrm{y}(0)=\left[\begin{array}{ll}0.5 & 0.2\end{array}\right]^{T}$

\section{CONCLUSION}

In this paper, we analyze nonlinear systems with time varying inputs. In particular, we show the effect of input variations on the trajectories of the transformed nonlinear systems in (10). We also show that the result obtained is useful to analysis and design of gain scheduling.

\section{References}

1. G. Stein, "Adaptive flight control-A pragmatic view," in Applications of Adaptive Control, New York: Academic Press, 1980.

2. M.J. Whatley and D.C. Pott, "Adaptive gain improves reactor control," Hydrocarbon Processing, pp. 75-78, May 1984.

3. J.S. Shamma and M. Athans, "Gain scheduling: Potential hazards and possible remedies," IEEE Control Syst. Mag., vol. 12, no. 3, pp. 101-107, 1992.

4. M. Kelemen, “A stability property," IEEE Trans. Automat. Contr., vol. AC-31, pp. 766-768, 1986.

5. J.S. Shamma and M. Athans, "Analysis of nonlinear gain scheduled control systems," IEEE Trans. Automat. Contr., vol. AC-35, pp. 898-907, 1990.

6. D.A. Lawrence and W.J. Rugh, "On a stability theorem for nonlinear systems with slowly varying inputs," IEEE Trans. Automat. Contr., vol. AC-35, pp. 860-864, 1990.

7. W.J. Rugh, "Analytical framework for gain scheduling," IEEE Control Syst. Mag., vol. 11, no. 1, pp. 79-84, 1991.

8. H.K. Khalil and P.V. Kokotovic, "On stability properties of nonlinear systems with slow-varying inputs," IEEE Trans. Automat. Contr., vol. AC-36, pp. 229, 1991. 


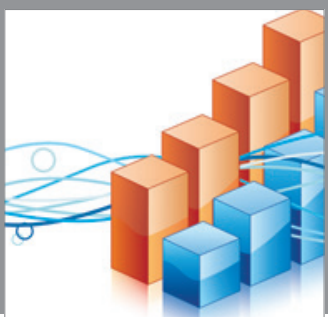

Advances in

Operations Research

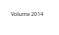

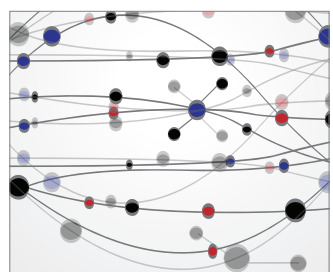

\section{The Scientific} World Journal
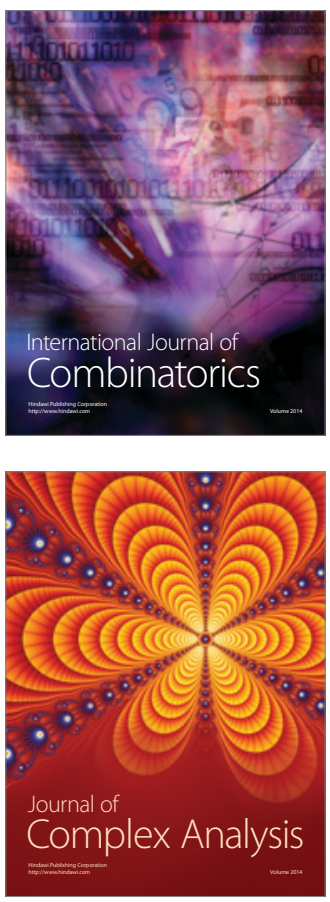

International Journal of

Mathematics and

Mathematical

Sciences
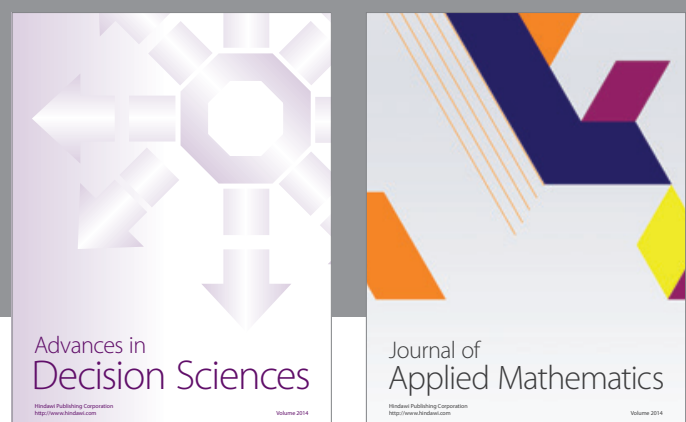

Journal of

Applied Mathematics
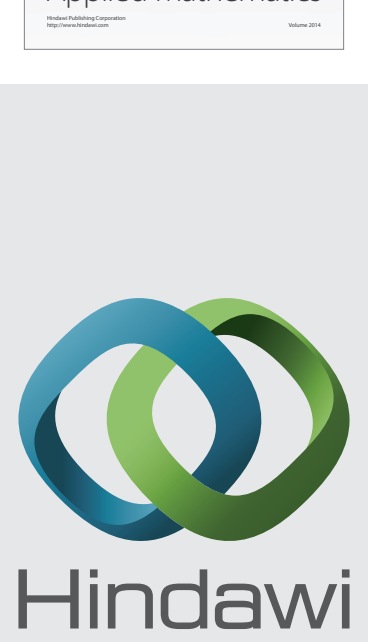

Submit your manuscripts at http://www.hindawi.com
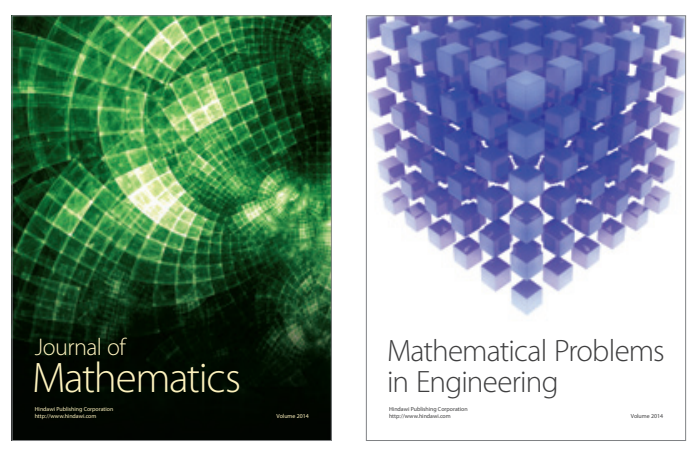

Mathematical Problems in Engineering
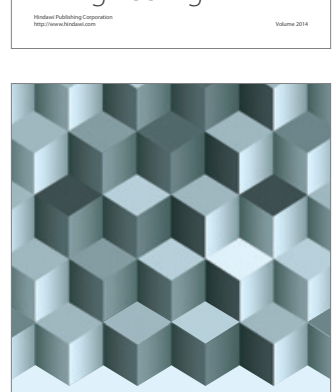

Journal of

Function Spaces
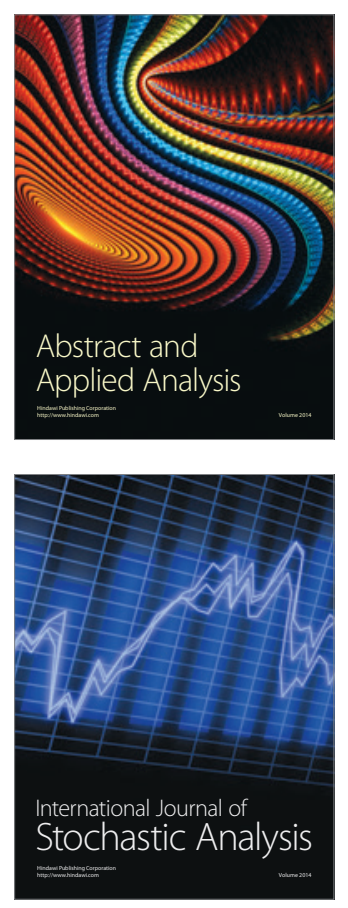

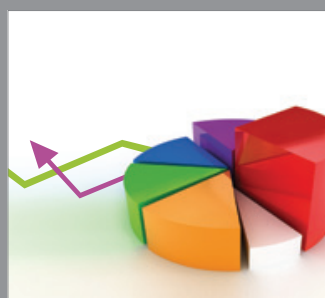

ournal of

Probability and Statistics

Promensencen
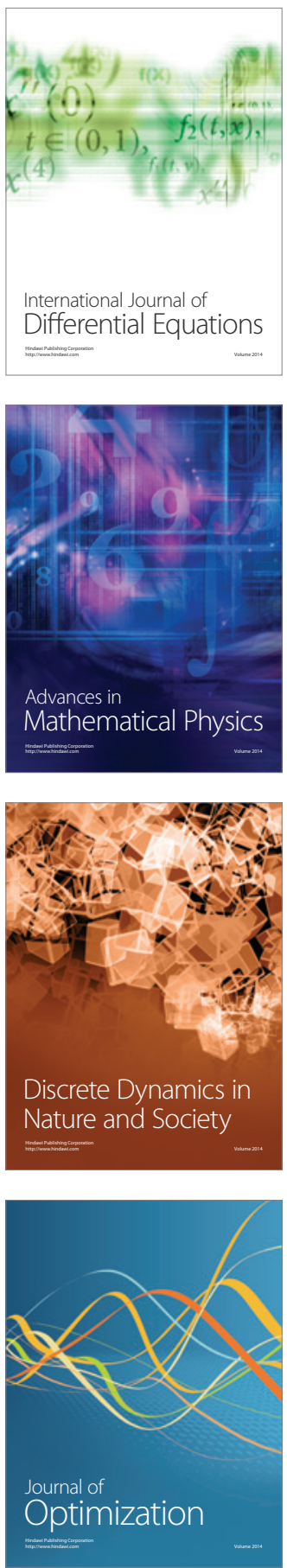